\title{
Quantum Criticality and Deconfinement in Phase Transitions Between Valence Bond Solids
}

\author{
Ashvin Vishwanath, ${ }^{1}$ L. Balents, ${ }^{2}$ and T. Senthil ${ }^{1}$ \\ ${ }^{1}$ Department of Physics, Massachusetts Institute of Technology, Cambridge MA 02139 \\ ${ }^{2}$ Department of Physics, University of California, Santa Barbara, CA 93106-4060.
}

(Dated: November 6, 2018)

\begin{abstract}
We consider spin-half quantum antiferromagnets in two spatial dimensions in the quantum limit, where the spins are in a valence bond solid (VBS) phase. The transitions between two such VBS phases is studied. In some cases, an interesting second order transition controlled by a fixed line with varying critical exponents is found. A specific example is provided by an antiferromagnetically coupled bilayer system on the honeycomb lattice where a continuous quantum phase transition can generically exist between two VBS phases. Furthermore, these critical points are deconfined, in the sense that gapped spin- $1 / 2$ spinon excitations emerge right at the transition. The low energy physics of this critical point (upto marginally irrelevant interactions) contains just a free quadratically dispersing 'photon'. The phase structure on one side of this continuous transition is very intricate consisting of a series of infinitely closely spaced further transitions in a 'devil's staircase' form. Analogies with previous examples of deconfined quantum criticality are emphasized. Closely related transitions in single layer systems are explored. These are second order only at some multicritical points. The solvable Rokshar-Kivelson point of quantum dimer models of single layer systems is found to correspond to a non-generic multicritical point
\end{abstract}

\section{INTRODUCTION}

Although the theoretical study of quantum phase transitions 1 has recieved much attention over the last several decades, it remains a source of rich and unexpected physics to this day. For example, recent work ${ }^{2.3}$ on the quantum criticality of certain quantum magnets (spin-1/2 quantum antiferromagnets on the square lattice) has shown that a direct and generically continuous transition between the Neel state and a valence bond solid state is possible. Such a transition violates 'Landau's rules' of classical phase transitions which prohibit a direct continuous transition between phases that posess such different symmetries. Perhaps more interestingly the critical theory is unusual and naturally expressed in terms of new emergent, 'deconfined' degrees of freedom that carry fractional spin, along with an emergent $U(1)$ gauge field. This is despite the absence (i.e confinement) of such fractional spin excitations or the associated gauge field in either phase. Such critical points were dubbed 'deconfined' quantum critical points (QCPs). In this paper we shall examine another example of a "deconfined" quantum critical point which occurs in a different context i.e. between two VBS phases.

The existence of such deconfined QCPs may be surprising since the gauge theories arising in condensed matter systems (apart from physical electromagnetism) are generally compact, i.e. are defined in terms of a periodic gauge connection $e^{i a}$ rather than a single valued noncompact vector potential $a$. In $\mathrm{D}=2+1$, such gauge theories are well known to be "always" (but see below) confining. In particular, the simplest and apparently generic compact $U(1)$ gauge theory hamiltonian is

$$
H=\sum_{r}\left\{\frac{1}{8 \pi \epsilon}|\mathbf{E}|^{2}+\frac{K}{2}|\boldsymbol{\Delta} \times \mathbf{E}|^{2}-\gamma \cos \boldsymbol{\Delta} \times \mathbf{a}\right\},
$$

with integer valued "electric field" $\mathbf{E}$ and $2 \pi$-periodic vector potential a (canonically conjugate to one another) defined on the links of a two-dimensional lattice. Here $\boldsymbol{\Delta}$ is the lattice gradient, and $\boldsymbol{\Delta} \times$ is the lattice curl, defined as the gauge flux through a plaquette. In the natural cases arising in condensed matter contexts, one imposes the Gauss law constraint $\boldsymbol{\Delta} \cdot \mathbf{E}=0$ (the "even" gauge theory) or $\boldsymbol{\Delta} \cdot \mathbf{E}_{r}=\epsilon_{r}$ on bipartite lattices, with $\epsilon_{r}= \pm 1$ taking opposite signs on the two sublattices (the "odd" gauge theory). In both cases, regardless of the values of $\gamma$ or the "dielectric constant" $0<\epsilon<\infty$, the gauge theory is always confining. The value of the nominally "irrelevant" (in the renormalization group sense) coupling $K$ is then immaterial.

Remarkably, Ref. 2 concluded that a deconfined phase is possible if a gauge theory similar to above is coupled to gapless matter fields, which arise naturally in quantum antiferromagnets at particular QCPs. In this paper we study a different class of transitions which provide other instances of 'deconfined' quantum criticality in quantum magnets. Although in contrast to Ref. 2, they are not examples of Landau forbidden transitions, they nevertheless display several interesting similarities to those priors. We consider transitions between two valence bond solid (VBS) states: quantum paramagnets with a gap to spin excitations. In either phase these spin-carrying excitations are conventional (though gapped) spin-1 magnons or their composites. We study direct second order transitions between two such phases where 'deconfinement' obtains right at the critical point. We show that the critical mode can be viewed as a gapless deconfined $U(1)$ gauge field with a quadratic dispersion. Moreover the magnon excitations, that are sharply defined gapped excitations on either side of the transition, break up into two weakly interacting (gapped) spin- $1 / 2$ spinons. These spinons are minimally coupled to the critical deconfined 
$U(1)$ gauge field. This results in a weak interaction between the spinons so that they are essentially free.

In contrast to the examples of deconfined quantum criticality of Ref. 2 which are strongly interacting 4 , the critical fixed points discussed here have a simple free field description. In particular, they may be considered as a limit of the compact gauge theory in Eq. (11) where the dielectric constant $\epsilon \rightarrow \infty$. At this point, the energy cost of an emergent electric flux to quadratic order in $\mathbf{E}$ is proportional to $|\boldsymbol{\Delta} \times \mathbf{E}|^{2}$ rather than $|\mathbf{E}|^{2}$ as usual. Despite the absence of gapless matter fields, this can provide a realization of a deconfined QCP, provided the system has sufficient (physically achievable) symmetries, as shown below. We emphasize that the appropriate physical symmetries occur naturally, for instance in an antiferromagnetically coupled spin- $1 / 2$ honeycomb bilayer. Moreover, the QCP in this case is "generic", i.e. only one physical parameter needs to be tuned (corresponding to tuning $1 / \epsilon$ through zero) to reach it. While particular "quantum dimer" models (see below) have provided some concrete realizations of compact $U(1)$ gauge theories, many other microscopic mechanisms leading to Eq. 1 exist, and it is fruitful to regard the gauge theory as the most general progenitor of such deconfined QCPs.

Closely related phenomena have been mentioned previously in the literature ${ }^{5.6}$ in the context of work on the quantum dimer mode $\mathbf{l}^{7}$. The dimer model provides a caricature of spin-gapped quantum paramagnetic phases and admits on certain lattices a solvable point known as the Rokhsar-Kivelson (RK) point. On a square or honeycomb lattice the RK point is known to be critical and to separate two phases with very different VBS order. An important development was the field theoretic description of this RK point, that was conjectured by Henley ${ }^{5}$, and further elaborated in ${ }^{6}$. In this paper we will analyze the generic behaviour expected in such a transition between VBS phases, and show that it differs in important ways from the physics of the RK point. In particular for single layer quantum spin systems on the square lattice we argue that generically there is no second order transition between two VBS phases. It has been argued in Ref. 8 that a similar situation also obtains for the single-layer honeycomb lattice, namely there is generically no second order transition. In contrast as we show below such a second order transition with 'deconfined' criticality obtains on the bilayer honeycomb lattice with antiferromagnetic interlayer coupling. In fact, a line of fixed points with continuously varying exponents is obtained. The universal long distance physics at one of these critical points corresponds to the long distance physics at the RK point (upto logarithmic corrections arising from a marginally irrelevant operator). Thus, although in general there are significant differences from the generic transition, the RK point in some cases can still provide useful information about the universal critical properties of these generic transitions. A unique feature of the RK point is that it occupies a very special place in the phase diagram of the generic spin model, a point that will be further discussed below.

We now summarize our results for the continuous quantum phase transition between two VBS phases of spin-1/2 quantum antiferromagnets on the bilayer honeycomb lattice. The precise nature of the neighbouring phases themselves will be described shortly. There is a simple Gaussian description of the critical theory which is parametrized by a 'stiffness' $K$ and has a dynamical scaling exponent $z=2$. In addition there is a marginally irrelevant operator that will lead to logarithmic corrections. Apart from the usual 'thermal' operator needed to tune to the critical point, the theory is found to have no other relevant perturbations for a range of stiffnesses, leading to a fixed line with continuously varying exponents depending upon $K$. Indeed, this is an interesting example of a fixed line in $D=2+1$ dimensions, and is closely related to three dimensional statistical physics models of the Lifshitz point in certain liquid crystal systems ${ }^{9}$.

Our results rely crucially on a 'dual' description of the quantum paramagnetic phases of antiferromagnets (with collinear spin correlations) in terms of a sine-Gordon (or 'height') field $\chi^{10,11}$. It is constructed (see Section III) by defining $E_{i}=\epsilon_{i j} \Delta_{j} \chi$, so that all physical properties are invariant under the global shift $\chi \rightarrow \chi+1$. This formulation may be obtained in a number of different ways which are briefly discussed in Section 11 below. In the continuum limit appropriate near critical points between various paramagnetic phases the Euclidean action of this model reads (for the honeycomb lattice bilayer system):

$$
\begin{aligned}
S & =S_{0}+S_{1}+S_{\text {inst }} \\
S_{0} & =\frac{1}{2} \int d^{2} x d \tau\left\{\left(\partial_{\tau} \chi\right)^{2}+\rho(\nabla \chi)^{2}+K\left(\nabla^{2} \chi\right)^{2}\right\}(3) \\
S_{1} & =\int d^{2} x d \tau \frac{u}{4}|\boldsymbol{\Delta} \chi|^{4}+\ldots \\
S_{\text {inst }} & =-\int d^{2} x d \tau \lambda \cos (2 \pi \chi)
\end{aligned}
$$

This continuum action is invariant under global integer shifts of the $\chi$ field, as well as $\chi \rightarrow-\chi(\mathbf{E} \rightarrow-\mathbf{E})$ which arises from symmetry under bilayer exchange. The ellipses represent other terms (higher derivatives and higher powers) which could be added that are consistent with the symmetries above. As discussed below, the field $\chi$ may be interpreted as the dual of a $U(1)$ gauge field. In this interpretation the $\lambda$ term describes instanton events. The transition of interest occurs when $\rho$ changes sign with $\rho=0$ at the critical point. Instantons are relevant in either phase but will turn out to be irrelevant at the critical point for some range of $K$. In addition the quartic term $u(\vec{\nabla} \chi)^{4}$ will be shown to be marginally irrelevant. Thus, the action $S_{0}$ in Eqn. 2 (which was recently studied along with several interesting generalizations in Ref ${ }^{12}$ ) will describe, upto marginally irrelevant terms, the fixed point action of a generic quantum critical point between two VBS phases of the bilayer honeycomb lattice antiferromagnet. When $\rho>0$ the sine Gordon field (also known 
as the height field) $\chi$ has zero 'tilt' in the ground state. It describes a featureless paramagnetic state of the original quantum magnet, which may be caricatured as being made of singlets formed on the interlayer rungs. However when $\rho<0$ one expects a finite 'tilt' i.e. $\chi_{r} \sim \mathbf{Q} \cdot \mathbf{r}$. Thus the transition may be expected to occur between a VBS phase with zero tilt of the height field and a VBS phase with a nonzero tilt of the height field - the tilt increasing 'continuously' from zero on moving away from the 'deconfined' critical point. Indeed while this general expectation is more or less correct, the detailed picture is somewhat more complicated owing to the relevance of the instanton operators away from the transition. In fact, as described in section $11 \mathrm{C}$ interesting structure obtains on the 'tilted' side of the transition. The background electric field (tilt) changes from its value of zero at the critical point to a non-zero value some distance away, through a fractal sequence of interleaving regions of constant tilt (confined phases) and non-constant incommensurate regions (deconfined phases) of zero width but finite total measure. The resulting structure is known as an 'incomplete devil's staircase' $\underline{8}$

In contrast to the above picture, the RK point sits between a VBS phase with zero tilt of the height field, and the staggered phase which has the maximum tilt to the height field, i.e. with $2 \pi \mathbf{Q}$ above one of the six reciprocal lattice vectors of minimum length. These phases are illustrated in Fig. 10 One can then ask whether the RK point occupies a special place in the phase diagram of the generic model. In fact, the well known property of the RK point that the ground state in each tilt (winding number) sector are all degenerate indicates that indeed it occupies a special place in the phase diagram. We have already noted that the RK point exhibits the same universal critical properties as the generic model after tuning a few relevant and marginal operators. However, to reach the special position occupied by the RK point in the phase diagram, fine tuning of several dangerously irrelevant operators is required. These operators do not affect critical properties but determine the phase structure in the vicinity of the critical point. The precise position of the RK point in the phase diagram is dicussed below. Note, the generic phase structure can be recovered if we add to the RK point an operator that (for example) corresponds to the quartic term $S_{1}$ in equation (2). Moreover, this particular operator will also generate logarithmic corrections to the RK point correlation functions, which will then precisely match the one of the generic critical points for the bilayer honeycomb system. Thus, generic behaviour, both in terms of critical properties as well as phase structure neighbouring the critical point can be obtained by adding this one operator to the RK point Hamiltonian.

In the usual quantum dimer model, this fine tuning of the Hamiltonian to access the RK point is achieved by keeping only terms that involve a single plaquette. However, if we regard the dimer model as an approximate description of some underlying quantum spin model, then in general we need to include dimer kinetic and potential terms on arbitrarily large-sized loops (but with coefficients that decrease with increasing loop size). The exact degeneracy mentioned above then obtains only if the dimer kinetic energy on every such loop is set equal to the corresponding potential energy. Thus for an underlying quantum spin model this exact degeneracy presumably requires 'infinite' fine-tuning.

A different way to draw a sharp distinction between the RK point and a generic (two parameter tuned) multicritical point that allows for a direct zero tilt to staggered (maximum tilt) state transition, is to ask - what phases can be accessed from these points by a small change in bare parameters? From the generic multicritical point (shown in Figure 2b) three states - the staggered, zero tilt and an infinitesimally tilted state - can be accessed. From the RK point however, in addition to the above three states, states with arbitrary values of the tilt can be accessed (Figure 2r). This is a consequence of the exact degeneracy of states with different tilts at the RK point which gives rise to the special phase structure in its vicinity.

The layout of this paper is as follows. In Section II we discuss the sine Gordon representation of this problem from different points of view, the non-linear sigma model approach of Haldane, the easy plane deformation of the spin half magnet of Lannert et al $\stackrel{13}{13}$, and the familiar height representation of the quantum dimer model. In Section [II] we analyze the spin half quantum antiferromagnet on the bilayer honeycomb lattice using this representation, and find a stable fixed line controlling the transitions. Various properties of these critical points are discussed, and the 'devil's staircase' phase structure is obtained. In section $\nabla$ the single layer square and honeycomb lattice spin-half antiferromagnet is discussed, where the generic transition between VBS phases is first order. In section VI we discuss the well known RK points, and how they fit into the general structure described in this paper.

\section{SINE GORDON REPRESENTATION FOR PARAMAGNETIC PHASES OF COLLINEAR QUANTUM ANTIFERROMAGNETS}

Our analysis relies crucially on a formulation ${ }^{10,11}$ of the physics of quantum paramagnetic phases in terms of a sine Gordon field theory on the dual lattice. In this Section, we describe this sine Gordon description and discuss its origin and interpretation from several different perspectives which together provide considerable insight. We will first discuss the single layer case and then move onto the double layer. The lattice Euclidean action for the sine Gordon model appropriate to a single layer has 
the following structure:

$$
\begin{aligned}
S & =S_{1}+S_{2}+\ldots \\
S_{1} & =\frac{1}{2} \int_{\tau} \sum_{r}\left(\partial_{\tau} \chi_{r}\right)^{2}+\rho(\Delta \chi)^{2}+K\left(\Delta^{2} \chi\right)^{2} \\
S_{2} & =-\int d \tau \sum_{r} \sum_{n=0}^{\infty} \lambda_{n} \cos \left(2 \pi n\left(\chi_{r}-\alpha_{r}\right)\right)
\end{aligned}
$$

Here $r$ runs over the sites of the dual lattice. The symbol $\boldsymbol{\Delta}$ refers to a lattice derivative. The ellipses represent other terms that are consistent with all the symmetries (both internal and lattice) that could be added. In the second term $n$ is an integer that runs from 0 to $\infty$. The $\alpha_{r}$ are independent of time but vary in a definite manner on spatial lattice sites. On the square lattice, $\alpha_{r}=0,1 / 4,1 / 2,3 / 4$ on four sublattices. Thus $\exp \left(2 i \pi \alpha_{r}\right)$ oscillates rapidly on four sublattices. For a spin model on the honeycomb lattice, the corresponding sine Gordon theory is defined on the dual triangular lattice. In this case $\alpha_{r}=0,1 / 3,2 / 3$ on the three sublattices of the triangular lattice so that $\exp \left(2 i \pi \alpha_{r}\right)$ oscillates rapidly on three sublattices.

Near the phase transitions of interest in this paper, the universal physics is adequately described by a continuum limit of this action. The oscillating phase factors due to the $\alpha_{r}$ means that in the continuum the only terms that survive from $S_{2}$ are those with $n=0(\bmod 4)$ for the square and $n=0(\bmod 3)$ for the honeycomb lattices respectively.

The physical basis of the sine Gordon description may be understood in many ways. We will mainly discuss the square lattice - the honeycomb lattice is very similar.

Gauge theoeretic/quantum dimer description: First consider a description of VBS phases in terms of quantum dimer models. A dimer is to be considered a caricature of a single valence bond and is taken to live on the bonds of the original lattice. For spin- $1 / 2$ systems, it is natural to constrain the dimer Hilbert space by requiring that there is exactly one dimer emanating out of each lattice site. It has long been appreciated that such quantum dimer models on bipartite lattices can be fruitfully viewed as compact $U(1)$ gauge theories. This is understood as follows: First divide the bipartite lattice under consideration into $A$ and $B$ sublattices. To each dimer we may associate an integer-valued vector 'electric field' $\mathbf{E}$ that starts from an $A$ sublattice point and ends at a $B$ sublattice point. In terms of these electric fields, the dimer constraint simply becomes the Gauss law

$$
\Delta \cdot \mathbf{E}= \pm 1
$$

where the + sign is for the $A$ sublattice and the - sign for the $B$ sublattice. Thus we see that the dimer Hilbert space is identical to that of a particular compact $U(1)$ gauge theory with fixed background 'charges' \pm 1 on the two sublattices. Such theories were christened 'odd gauge theories' in Ref. 6 .
Alternately such a gauge theoretic description of the disordered phases of the antiferromagnet can be directly obtained by starting with a slave particle (eg. Schwinger boson or fermion) description. Consider for instance a Schwinger boson representation. This has a $U(1)$ gauge redundancy associated with arbitrary phase rotations of the bosons at each site. In a spin gapped paramagnetic phase the Schwinger bosons can be integrated out and the physics is described by a compact $U(1)$ gauge theory. In this route too an odd gauge theory obtains.

There is a well-known duality mapping between compact $U(1)$ gauge theories and sine-Gordon field theories - see for instance Ref. 14. The interpretation of the sine Gordon field is as follows. In the absence of the compactness in the gauge theory, the total magnetic flux is exactly conserved. There is a corresponding (topological) global $U(1)$ symmetry. In the sine-Gordon representation this becomes an ordinary global $U(1)$ symmetry that corresponds to an arbitrary global shift of the sine-Gordon field. This symmetry is present in the sine Gordon model if the coefficient $\lambda_{n}$ of all the cosine terms is set to zero. Including compactness in the original gauge theory allows for 'instanton' events which destroy flux conservation. Indeed the flux can change in multiples of $2 \pi$. These are precisely captured by including terms like $\cos 2 \pi n\left(\chi_{r}-\alpha_{r}\right)$ in the sine Gordon description. Thus $e^{2 \pi i \chi}$ corresponds to an instanton event at which $2 \pi$ units of gauge flux is created. The shift $\alpha_{r}$ is due to the 'oddness' of the gauge theory. Specifically the presence of background charges in the gauge theory leads to Aharanov-Bohm phases for the gauge flux which are encapsulated in the shifts $\alpha_{r}$ - for details see Ref $f^{15}$.

Semiclassical Description: Much further insight is obtained by a semiclassical perspective that is particularly appropriate if there is considerable short-ranged Neel order. Deep in the Neel phase the long distance low energy fluctuations of the Neel vector are described by the familiar quantum $O(3)$ non-linear sigma model field theory. To correctly describe quantum paramagnetic phases it has been recognized for some time now that this continuum field theory must be augmented by appropriate Berry phase terms that are sensitive to the microscopic spin at each lattice site and to the details of the lattice structure. The Berry phase terms vanish for all smooth configurations of the Neel field. In two spatial dimensions note that such smooth configurations allow for topologically non-trivial configurations known as skyrmions. However as shown by Haldane the Berry phases are non-zero in the presence of singular configurations -known as hedgehogs or monopoles - in space-time. At the location of the monopole the skyrmion number associated with the Neel field configuration changes. That such skyrmion tunneling events are allowed is a consequence of the presence of a lattice in the microscopic spin model. The calculation of Ref. 16 shows that the Berry phases associated with a single monopole (defined on the plaquettes of the original lattice) oscillates from one plaquette to another with amplitude $\exp 2 i \pi \alpha_{r}$ where $\alpha_{r}$ is 
as defined above on the dual lattice sites (or equivalently on the plaquettes of the original lattice).

The quantum paramagnetic state is associated with a proliferation of such monopole events. The Berry phases associated with a single monopole event leads to it transforming non-trivially under lattice symmetry operations. Thus proliferation of single-strength monopoles leads to broken lattice symmetry in the paramagnetic state.

To describe the different possible paramagnetic phases, it is convenient to imagine integrating out all gapped spin-carrying excitations and focus on the physics of the skyrmion fields and the associated monopole events. First consider a limit in which the monopoles are ignored (i.e imagine tuning the monopole fugacity to zero). In this limit the skyrmion number is exactly conserved. This corresponds to a hidden (topological) global $U(1)$ symmetry in the absence of monopoles. The paramagnetic phase may then be thought of as a condensate of these skyrmions so that this global $U(1)$ symmetry is spontaneously broken. The low energy excitations are fluctuations of the phase of the skyrmion field and will be gapless. Indeed the corresponding action may be identified with $S_{1}$ above with $\chi / 2 \pi$ being the phase of the skyrmion field. Including monopole events leads to explicit breaking of this global $U(1)$ symmetry. Clearly in this picture the $S_{2}$ term corresponds precisely to skyrmion creation events (which are the monopoles) with the appropriate Haldane oscillating phases encapsulated in the shift fields $\alpha_{r}$.

Easy Plane Limit: Finally we briefly mention a derivation of the action above in the easy plane limit of the original spin model. As argued in Ref 13.17 (see Ref,$\stackrel{2}{,}$ for a physical discussion), a very useful continuum description of easy plane spin- $1 / 2$ magnets is provided by focusing on vortex fields (known as 'merons') in the (XY-like) order parameter. The dual action takes the form

$$
\begin{aligned}
\mathcal{L} & =\mathcal{L}_{\psi}+\mathcal{L}_{v}+\mathcal{L}_{A}+\mathcal{L}_{\text {inst }} \\
\mathcal{L}_{\psi} & =\sum_{a=1,2}\left|\left(\partial_{\mu}-i A_{\mu}\right) \psi_{a}\right|^{2}+r|\psi|^{2}+u\left(|\psi|^{2}\right)^{2}(11) \\
\mathcal{L}_{v} & =v\left|\psi_{1}\right|^{2}\left|\psi_{2}\right|^{2} \\
\mathcal{L}_{A} & =\kappa\left(\epsilon_{\mu \nu \kappa} \partial_{\nu} A_{\kappa}\right)^{2} \\
\mathcal{L}_{\text {inst }} & =-\lambda_{4}\left(\left(\psi_{1}^{*} \psi_{2}\right)^{4}+\text { c.c }\right) .
\end{aligned}
$$

Here $\psi_{1,2}$ represent the two meron fields that are minimally coupled ( as is usual) to a non-compact $U(1)$ gauge field $\vec{A}$, and $|\psi|^{2} \equiv\left|\psi_{1}\right|^{2}+\left|\psi_{2}\right|^{2}$. As described in Ref. [2 for the square lattice the last term physically describes the monopole or instanton events discussed above for the isotropic models. This continuum model has a global $Z_{4}$ symmetry associated with $\pm(\pi / 4+m \pi / 2)$ shifts of the phase of $\psi_{1}$ and $\psi_{2}$ respectively $(m=0,1,2,3)$. In this description valence bond solid phases correspond to $\left\langle\psi_{1}\right\rangle=\left\langle\psi_{2}\right\rangle \neq 0$. In such a condensate, the gauge field $\vec{A}$ acquires a mass by the usual Anderson-Higgs mechanism and may be ignored at low energies. Furthermore the global $Z_{4}$ symmetry is also broken - thus a low energy description is provided by focusing on the relative phase $\theta$ between $\psi_{1}$ and $\psi_{2}$. Clearly the theory has the same structure as the continuum limit of Eqn. [6 and we identify $\chi=2 \pi \theta$. This discussion readily generalizes to the honeycomb lattice - the main difference is that the monopole events are tripled (leading to $Z_{3}$ symmetry).

¿From any one of these perspectives it is clear that (anti)vortices in the $\chi$ field correspond to spin- $1 / 2$ spinon configurations in the original spin model. Specifically we define a (anti)vortex as a point in space around which

$$
\int d \mathbf{l} \cdot \nabla \chi= \pm 1
$$

For instance in the dimer model these correspond precisely to points where the dimer constraint is violated (i.e to monomers). Equivalently we note that skyrmions and spinons see each other ${ }^{18}$ as sources of $2 \pi$ flux - so that a spinon configuration corresponds to a vortex in the skyrmion phase.

Bilayer Systems: The discussion above is readily adapted to bilayer systems. Specifically consider a bilayer spin-1/2 quantum antiferromagnet on a square or honeycomb lattice. The symmetries of the microscopic Hamiltonian now include the Ising-like layer exchange symmetry in addition to $S U(2)$ spin rotation, time reversal and all the lattice symmetries. This layer exchange symmetry will play an important role in our analysis. Consider first the limit in which the interlayer antiferromagnetic exchange on each rung is the largest coupling. In this limit it is appropriate to first diagonalize the 'rung' Hamiltonian. For each rung, the ground state is a singlet and the first excited state is a triplet. A useful model of such a bilayer is to replace each rung by an $O(3)$ quantum rotor with the Hamiltonian

$$
H=\frac{g}{2} \sum_{i} \vec{L}_{i}^{2}-J \sum_{<i j>} \hat{n}_{i} \cdot \hat{n}_{j}+\ldots .
$$

Here $\hat{n}_{i}$ is a unit three component vector defined on each rung (labelled by i) and $\vec{L}_{i}$ is the corresponding angular momentum. The ellipses represent other short-ranged terms consistent with the symmetries. The rotor vector $\hat{n}_{i}$ and the angular momentum $\vec{L}_{i}$ have the same symmetry properties as the difference and sum of the two microscopic spins on the rung at $i$ respectively. Under layer exchange we then have

$$
\begin{aligned}
& \hat{n}_{i} \rightarrow-\hat{n}_{i} \\
& \vec{L}_{i} \rightarrow \vec{L}_{i}
\end{aligned}
$$

Thus layer exchange symmetry implies that the rotor Hamiltonian be invariant under the full group $O(3)$ of rotations (which includes improper rotations). In addition time reversal is a separate symmetry that is implemented by an antinunitary operator that changes the sign of both $\hat{n}$ and $\vec{L}$. We remark that this must be contrasted with single layer Heisenberg spin magnets. These can 
also be modelled as quantum rotors but with appropriate monopoles placed at the origin of the $\hat{n}$-sphere at each site. Now the presence of the monopoles implies that the improper rotations of the rotor vector are no longer symmetries. Thus these must be regarded as $S O(3)$ rotors. This distinction will also be important for us below.

Consider now paramagnetic phases of the bilayer model. We will specifically be interested in phases that obtain close to the strong interlayer exchange limit where the $O(3)$ rotor description becomes appropriate. As with the single layer systems discussed above it will be convenient to obtain a gauge theoretic description of these paramagnetic phases. This may be obtained by passing to a $C P^{1}$ description of the rotors in terms of spinon variables $z$. The $z$ fields are minimally coupled to a compact $U(1)$ gauge field $\vec{a}$ but unlike the single layer case the mean spinon number is zero per site. In a mean field description of paramagnetic phases the spinon fields will be gapped. Beyond mean field, integrating out the gapped spinons leads to a compact $U(1)$ gauge theory. The ultimate fate of the spinons is determined by whether or not this gauge theory is confined. Again in contrast to the single layer case the Gauss law constraint of this gauge theory is simply

$$
\Delta \cdot \mathbf{E}=0
$$

with no background charges. Here $E_{i j}$ is the 'electric' field defined on the links of the honeycomb or square lattice. As usual this is conjugate to the gauge field $a_{i j}$ :

$$
\left[a_{i j}, E_{i j}\right]=i \text {. }
$$

What are the symmetries of this gauge theory? Clearly all the symmetries of the square or honeycomb lattice that forms each layer are also symmetries of the gauge theory. In addition the symmetry of the rotors under improper rotations (the layer exchange symmetry) implies that the gauge theory must be invariant under the discrete symmetry

$$
\begin{aligned}
E_{i j} & \rightarrow-E_{i j} \\
a_{i j} & \rightarrow-a_{i j}
\end{aligned}
$$

This may be seen in several ways. For instance we note that the magnetic field corresponding to the gauge field $a$ is precisely the skyrmion density associated with the configuration of the $\hat{n}$ fields. The latter is odd under improper rotations of $\hat{n}$ (for instance there is a well-known expression for the skyrmion density as a trilinear in $\hat{n}$ ). Similarly the electric fields correspond to the skyrmion currents which are likewise odd under improper rotations. Parenthetically we note that under (the antiunitary) time reversal the electric field is even while the gauge field is odd.

This compact $U(1)$ gauge theory is readily dualized to obtain a dual sine-Gordon description in terms of the $\chi$ field. The dual action takes the form

$$
\begin{aligned}
S & =S_{1}+S_{2}+\ldots \\
S_{1} & =\frac{1}{2} \int_{\tau} \sum_{r}\left(\partial_{\tau} \chi_{r}\right)^{2}+\rho(\Delta \chi)^{2}+K\left(\Delta^{2} \chi\right)^{2} \\
S_{2} & =-\int d \tau \sum_{r} \sum_{n=1}^{\infty} \lambda_{n} \cos \left(2 \pi n \chi_{r}\right)
\end{aligned}
$$

There are two important differences with the single layer case. First the absence of background charges in the gauge theory implies that there are no offsets $\alpha_{r}$ for the height fields $\chi_{r}$. Second the discrete layer exchange symmetry implies that the action must be invariant under $\chi_{r} \rightarrow-\chi_{r}$.

It is interesting to contrast the bilayer with a spin$3 / 2$ antiferromagnet in a single honeycomb layer. In the latter the gauge theory appropriate to the paramagnetic phase may be viewed as an 'even' gauge theory, i.e one where there are no background charges in the Gauss law constraint. But nevertheless as the microscopic model is not invariant under improper rotations of the spin (or equivalently the rotor vector in a rotor description) the gauge theory does not have the discrete symmetry of Eqn. 21 associated with changing the signs of both $E$ and $a$.

\section{THE BILAYER HONEYCOMB LATTICE}

In this Section, we specialize to the bilayer honeycomb lattice, assuming the presence of strong interlayer antiferromagnetic coupling. We will consider the lattice valence bond solid phases and phase transitions of this spin half quantum antiferromagnet using the sine Gordon description Eqn. 23] It will be convenient to explicitly write out the lowest order non-linear terms that are allowed by the symmetries. These take the form

$$
S_{\text {int }}=\int d \tau \frac{u}{4}|\Delta \chi|^{4}+\frac{v}{6} \operatorname{Re}\left[\left(\Delta_{x}+i \Delta_{y}\right) \chi\right]^{6}
$$

so that the full action is

$$
S=S_{1}+S_{2}+S_{\text {int }}
$$

with $S_{1,2}$ given in Eqn. 6] We have included in $S_{\text {int }}$ the $v$ term which gives the lowest order effect of the sixfold broken rotational symmetry of the hexagonal lattice. Note that it appears only at sixth order in $\Delta \chi$, and hence is nominally irrelevant at the critical point. It is nevertheless important for $\rho<0$ (see below). Note that the symmetries of the bilayer system include $\chi_{r} \rightarrow-\chi_{r}$ at each site $r$ which corresponds to layer exchange. This symmetry forbids the appearance of terms that are odd in $\chi$. Such terms are allowed in single layer systems that do not have this symmetry - indeed Ref. 8 identifies a cubic term that drives the transition first order.

Let us for a moment consider how the various VBS phases arise from the action (27). For $\rho>0$, the system 


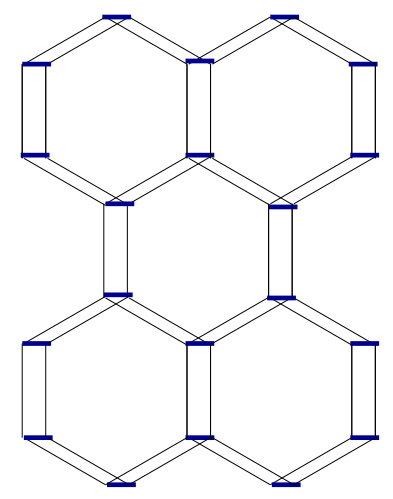

(a)

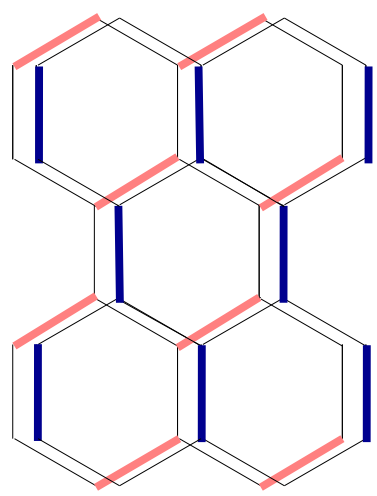

(b)
FIG. 1: Caricature of VBS phases on the bilayer honeycomb lattice. (a) The zero tilt state, with singlet bonds (thick lines) on the interlayer rungs. Note, this state does not break any lattice symmetry. (b) One of six possible maximally tilted (staggered) phases.

would like to have zero tilt $\boldsymbol{\Delta} \chi$ on the average. Moreover, we can ask what the effect of the monopole tunneling term is, the first such term is the one that inserts a single monopoles $(n=1)$ of Eqn 25] and it is easily seen that this operator has long ranged correlations if $\rho \neq 0$, and is hence a relevant perturbation. The resulting phase will be one where the height field is pinned at a uniform value, and this state may be caricatured as one where the spins form singlets with their partners in the other layer as shown in Fig. (19).

For negative values of $\rho$, a state with a finite tilt is expected, i.e.

$$
\mathbf{k}=\langle 2 \pi \Delta \chi\rangle \neq 0 .
$$

Ignoring the monopole operators for the moment, the system will choose a tilt $\mathbf{k}_{0}$ whose magnitude for small negative $\rho$ is obtained at the mean-field level by minimizing together the $\rho$ and $u$ terms, i.e.

$$
\left|\mathbf{k}_{0}\right|=2 \pi \sqrt{\frac{-\rho}{u}}
$$

with corrections of $O\left[v / u(-\rho / u)^{3 / 2}\right]$ from $v$ and other higher order terms. Fluctuation effects due to the marginality of $u$ will slightly enhance $\left|\mathbf{k}_{0}\right|$ by a logarithmic factor of little importance. The direction of the vector $\mathbf{k}_{0}$ is, however, determined by the sign of $v$. In particular, the six discrete directions with $k_{0 x}+i k_{0 y}=$ $\left|\mathbf{k}_{0}\right| e^{i 2 \pi m / 6}$ or $k_{0 x}+i k_{0 y}=\left|\mathbf{k}_{0}\right| e^{i 2 \pi(m+1 / 2) / 6}$, with $m=$ $0 \ldots 5$ are preferred for $v<0, v>0$, respectively. The added effects of monopoles, which will modify the true tilt vector to $\mathbf{k} \neq \mathbf{k}_{0}$, however, will have to be carefully considered discussed in the last part of this section.

In any case, a phase transition between a VBS with zero average tilt, and one with nonzero average tilt, cor- responds to taking $\rho$ from positive to negative values. The phase transition that lies between requires that we look at the theory with $\rho=0$.

\section{A. Stability of the Fixed Line Controlling the Transition}

The critical theory for such a VBS transition is then proposed to be:

$$
S_{c}=\int d \tau \sum_{r} \frac{1}{2}\left\{\left(\partial_{\tau} \chi\right)^{2}+K\left(\Delta^{2} \chi\right)^{2}\right\}
$$

we need to check that this simple Gaussian theory is stable against switching on a small monopole tunneling (term $S_{2}$ in the action (23) ) and quartic interactions $\left(S_{\text {int }}\right.$ of equation (26) ). Again, we consider correlators of the single monopole tunneling event, the $n=1$ term. This now has the following power law decay in space:

$$
\left\langle e^{i 2 \pi \chi_{r}(0)} e^{-i 2 \pi \chi_{0}(0)}\right\rangle \sim \frac{1}{r^{\frac{\pi}{\sqrt{K}}}}
$$

which implies that the monopole insertion operator is irrelevant if $\frac{\pi}{2 \sqrt{K}}>4$, or equivalently $0<K<\left(\frac{\pi}{8}\right)^{2}$. Thus, there is a line of fixed points, with different exponents, that are parametrized by $K$ and are all stable against switching on weak monopole tunneling. Indeed this is very similar to the line of fixed points obtained in $D=1+1$ in a variety of systems such as the spin half XXZ chain. Note however that our theory has dynamical exponent $z=2$.

We now consider the effect of the quartic interaction term, $S_{\text {int }}$ in Eqn. 26] which by naive power counting is marginal at these fixed points. Since we will be looking at values of $K$ for which the monopole tunneling events are irrelevant, they are disregarded in the discussion below. We consider a continuum model of the critical theory (30), with modes restricted to wavevectors below a certain cutoff $\Lambda$, We perform a one loop RG, assuming that we start with a small value of the interaction parameter $u$, and study its flow on integrating out the large wave-vector modes $\Lambda(1-d l)<|k|<\Lambda$. After the appropriate rescaling to keep the quadratic term (30) invariant, we obtain the following flow equation for the quartic coupling 9 :

$$
\frac{d u}{d l}=-\frac{9}{16 \pi K^{\frac{3}{2}}} u^{2} .
$$

This implies that a quartic coupling with $u>0$ is marginally irrelevant, and the coupling flows back to zero logarithmically with distance. Therefore the long distance physics in this case will be controlled by the critical action (30), with logarithmic corrections arising from this marginally irrelevant operator. Thus the critical points are stable towards turning on a quartic interaction for $u>0$. (For $u<0$ however, the quartic coupling is relevant, and the transition is very likely driven first order 
- as is indeed already the case in mean field theory for negative $u$ ).

\section{B. Properties of the Transition}

The analysis above has established the presence of a fixed line controlling the transition between VBS phases with zero tilt and those with a non-zero tilt of the height field. In this subsection, we discuss some properties of this fixed line. We first note that the irrelevance of the monopole tunneling terms implies that the global symmetry of the continuum theory is enlarged to $U(1)$. This corresponds to invariance under arbitrary global shifts of the height field $\chi$. In terms of the $U(1)$ gauge theory (whose dual is the sine Gordon theory) this implies that the compactness is asymptotically irrelevant all along this fixed line. Indeed as shown in Appendix $\mathrm{A}$ the free Gaussian action is readily seen to describe a quadratically dispersing gapless photon in the gauge theory representation. The gauge flux of the theory is then conserved. This signals deconfinement. However the monopoles are important to correctly describe the physics of the phases on either side of the transition - thus they represent 'dangerously irrelevant' perturbations.

If we ignore the marginally irrelevant quartic term the fixed point theory has a free field form. Thus all aspects of the critical behavior while non-trivial are eminently tractable. For instance it should be possible to compute real time dynamical correlators of, say, the operator $e^{i 2 \pi \chi_{r}(t)}$ at non-zero temperatures in the scaling limit. We will however not pursue this here.

It is instructive to ask about the fate of the gapped spin-carrying excitations right at this critical fixed line. As mentioned in Section III the presence of a spin- $1 / 2$ spinon at some spatial site leads to a vortex in the height field $\chi$. Away from the critical point, in (for instance) the zero tilt VBS, the relevance of the monople tunneling terms lead to pinning of the height field which implies that there is a huge energy cost that increases linearly with system size for these vortices. More precisely consider a pair of spinons (on opposite sublattices of the original lattice). This generates a vortex-antivortex pair in $\chi$. The energy cost for separating this pair by a distance $R$ grows linearly with $R$ away from the critical point. Thus we have (linear) confinement of spinons and the elementary spin-carrying excitations have spin-1. But right at the critical fixed line the enlargement of the symmetry to $U(1)$ implies that vortices in the $\chi$ field are cheap. Ignoring the quartic perturbation, an elementary computation shows that the energy cost of a vortex is finite independent of system size. Including the quartic term leads to a weak $1 / R^{2}$ interaction (up to logarithmic corrections due to the marginal irrelevance of $u$ ) between a vortexantivortex pair separated by distance $R$ - this then is the interaction between two spinons (on two opposite sublattices) separated by a distance $R$. Thus as expected the spinons are deconfined and free to propagate above a spin gap.

Within the approximation of ignoring the weak interaction between the two spinons, the magnon spectral function $A(\vec{k}, \omega)$ at the gap edge is readily calculated. One finds a sharp step $A(\vec{k}, \omega) \sim \theta(\omega-\Delta)$ where $\Delta$ is the spin gap. Thus the magnon spectral function has no quasiparticle peak and is anomalously broad.

\section{Devil's Staircase}

We now consider the behavior on the "tilted" side of the Lifshitz point, in which the compact gauge theory is expected to have a non-vanishing background electric field. The neighborhood of the Lifshitz point has been argued in Ref 8 to realize an "incomplete Devil's staircase". While the essential features have already been sketched in Ref. 8, we recapitulate them here for completeness and to present a few additional points not mentioned therein. In particular, we will describe the thermal transitions of the commensurate tilted VBS states within the devil's staircase, and point out two distinct types of low energy excitations within these phases.

Let us first think generally about the nature of phases with some finite and non-zero background electric field, i.e. not in the direct vicinity of the putative critical point. At this stage we do not take any continuum limit, working with a field $\chi_{r}$ defined on the discrete lattice sites (of the dual square or hexagonal lattices). If one neglects at first the compactness of the gauge theory, i.e. the terms breaking continuous translational symmetry of $\chi_{r}$, then one may write $\chi_{r}=\mathbf{k}_{0} \cdot \mathbf{r} / 2 \pi+\delta \chi_{r}$, with $\delta \chi_{r}$ describing fluctuations around the putative average tilt $\mathbf{k}_{0}$ determined as in Sec. III by minimizing with respect to $\mathbf{k}_{0}$ the non-monopole terms in the action. One should regard $\mathbf{k}_{0}$ as the continuously varying "tilt" the system would have were there no cosine (monopole) terms. We will see that the true tilt, i.e. $\langle\boldsymbol{\Delta} \chi\rangle=\mathbf{k}$, is close but not generally equal to $\mathbf{k}_{0}$. The fluctuations of $\delta \chi_{r}$ will then be described by a theory of the form

$$
\begin{aligned}
S_{t i l t}= & \int d \tau \sum_{r} \frac{1}{2}\left\{\left|\partial_{\tau} \delta \chi_{r}\right|^{2}+\tilde{\rho}\left|\boldsymbol{\Delta} \delta \chi_{r}\right|^{2}\right\} \\
& -\sum_{n} \tilde{\lambda}_{n} \cos \left[2 \pi n \delta \chi_{r}+n \mathbf{k}_{0} \cdot \mathbf{r}\right],
\end{aligned}
$$

Note that, unlike at the critical point, the fluctuations of $\delta \chi_{r}$ at the quadratic level have a non-vanishing (renormalized) stiffness $\tilde{\rho}$. Hence the fluctuations of $\delta \chi_{r}$ will be bounded, and any non-oscillatory cosine term breaking the continuous translational symmetry of $\delta \chi_{r}$ will "pin" it, however weak. This pinning corresponds to confinement in the original gauge theory, and a VBS phase in the dimer model. We note in passing that, actually, depending upon the value of $\mathbf{k}_{0}$ and anisotropies in the original action, $\tilde{\rho}$ can be replaced by a more general tensor. Again, this complication does not modify any of the qualitative results of this section, and so will be ignored. 
For a "generic" value of $\mathbf{k}_{0}$ all the cosines oscillate since $\mathbf{k}_{0} \cdot \mathbf{r}$ will be an irrational multiple of $2 \pi$. There are, however, an infinite dense set of values of $\mathbf{k}$ for which $\mathbf{k} \cdot \mathbf{r}$ is a rational multiple of $2 \pi$ (for all $\mathbf{r}$ ). In this case, there will be some minimal value of $n$ for which the $\lambda_{n}$ term does not oscillate (for $\delta \chi_{r}=0$ ). Clearly, at these special values of $\mathbf{k}$, this cosine term is relevant and the system is in some confined VBS phase. Furthermore, any irrational $\mathbf{k}_{0}$ is arbitrarily close to one of these rational $\mathbf{k}$ values, so that, although the cosines in general oscillate on the lattice, some of them oscillate extremely slowly. Since in general the cosines are not infinitesimally weak (i.e. the $\lambda_{n}$ are finite and non-zero), a sufficiently long wavelength oscillation of the cosine term could potentially "pin" the $\delta \chi_{r}$ field even in such cases. To see whether this occurs, let us suppose the $n^{\text {th }}$ cosine term oscillates weakly, i.e. $e^{i n \mathbf{k}_{0} \cdot \mathbf{r}}=e^{i n \delta \mathbf{k} \cdot \mathbf{r}}$ for all lattice vectors $\mathbf{r}$, with $n|\delta \mathbf{k}| \ll 2 \pi$. Then, keeping only this cosine term, we have approximately

$$
\begin{aligned}
& S_{\text {tilt }}=\int d \tau \sum_{r} \frac{1}{2}\left\{\left|\partial_{\tau} \delta \chi_{r}\right|^{2}+\tilde{\rho}\left|\boldsymbol{\Delta} \delta \chi_{r}\right|^{2}\right\} \\
& -\tilde{\lambda}_{n} \cos \left[2 \pi n \delta \chi_{r}+n \delta \mathbf{k} \cdot \mathbf{r}\right] .
\end{aligned}
$$

Since the fluctuations of $\delta \chi_{r}$ are bounded, we may estimate the effects of the cosine term by ignoring these fluctuations and minimizing the action. The minimal action configurations are clearly constant in imaginary time, $\partial_{\tau} \delta \chi_{r}=0$. Roughly, then, the field $\delta \chi_{r}$ can either be constant, minimizing the stiffness term but gaining no (lowering of the) energy from the cosine, or it can choose to tilt slightly to take advantage of the cosine term, costing some energy from the stiffness. Dimensionally, the latter will be favorable if $\tilde{\lambda}_{n} \gtrsim \tilde{\rho}|\delta \mathbf{k}|^{2}$. Now $|\delta \mathbf{k}|$ can be made arbitrarily small by increasing $n$ (typically decreasing as $1 / n)$, so this inequality will be satisfied if the $\tilde{\lambda}_{n}$ do not decrease too rapidly (i.e. faster than $1 / n^{2}$ ) with $n$. However, it is perfectly conceivable that the $\tilde{\lambda}_{n}$ terms do decrease faster than $1 / n^{2}$, and in this circumstance, there will be incommensurate values of $\mathbf{k}_{0}$ for which the $\chi$ field remains unpinned, and all monopole terms remain irrelevant. This has been argued to be the case in the immediate neighborhood of RK points in Ref. 8 . Note that even so, there are commensurate pinned states arbitrarily close to this incommensurate state. The selfsimilar succession of various commensurate and incommensurate states is the incomplete Devil's staircase mentioned above. The term "incomplete" indicates that the incommensurate unpinned regions exist (and have finite measure, as can also be argued).

The same arguments apply to the neighborhood of the VBS transitions discussed here, and we will sketch the reasoning in order to make a few more observations. Note that in these cases (e.g. for the honeycomb bilayer), the Lifshitz point itself is generic, i.e. can be potentially observed in a physical system by varying only one parameter.

First, we comment on a minor subtlety. A naïve anal- ysis of the continuum field theory, Eq2 would suggest that on the tilted side of the Lifshitz point, the tilt increases smoothly from zero. This appears to be the case since at the Lifshitz point, all cosine (monopole) operators are irrelevant. One the tilted side of the transition, in fact the tilt does not increase smoothly, but in the staircase fashion. This occurs because irrelevant cosine operators at the Lifshitz point become relevant on the tilted side of the "transition", i.e. these operators are dangerously irrelevant in renormalization group parlance. In fact, the naïve smooth increase in slope (background electric field of the gauge theory) occurring on this side of the transition is replaced by a slope which contains piecewise constant and incommensurate regions, which become more and more closely spaced as the Lifshitz point is approached, forming an infinite sequence that approximates the naive continuous curve (of e.g. $|\mathbf{k}|$ versus $\rho$ ) arbitrary well if one looks arbitrarily close to the Lifshitz point.

A full description of the devil's staircase is beyond the scope of this paper. It is instructive and indicative of the general structure to consider some simple "families" of plateaus in the tilt $\mathbf{k}$ that obtain near the critical point. In general, the condition for a plateau is that $\cos (n \mathbf{k} \cdot \mathbf{r})$ does not oscillate on the dual hexagonal lattice. This condition is equivalent to requiring that $n \mathbf{k}$ is a reciprocal lattice vector of the hexagonal lattice. An arbitrary reciprocal lattice vector can be written as $n_{1} \mathbf{b}_{1}+n_{2} \mathbf{b}_{2}$, with $\mathbf{b}_{1}=(2 \pi, 2 \pi / \sqrt{3}), \mathbf{b}_{2}=(0,-4 \pi / \sqrt{3})$. Hence, the condition on $\mathbf{k}$ is

$$
\vec{k}=\left(\frac{n_{1}}{n}\right) \mathbf{b}_{1}+\left(\frac{n_{2}}{n}\right) \mathbf{b}_{2}
$$

where $n, n_{1}, n_{2}$ are integers, and the pairs $n_{1}, n$ and $n_{2}, n$ can be taken to be relatively prime. The "strongest" such commensurate tilts are those with minimal $n$, since these correspond to $n$-monopole events, which become less relevant as $n$ increases.

In understanding the behavior for small $\mathbf{k}_{0}$, we need to investigate those commensurate tilts for which $\mathbf{k}$ is small but non-zero. Clearly for Eq. 35 this will occur for large $n$. Since the two vectors $\mathbf{b}_{1}, \mathbf{b}_{2}$ are linearly independent, the coefficients of both must be small for $\mathbf{k}$ to be small. Since the two numerators in these coefficients are integers, for a given small magnitude $|k| \ll 1$, one clearly then needs at least $n \gtrsim|k|^{-1} \gg 1$. Larger values of $n$ can also yield the same $|k|$, by increasing the numerators accordingly. However, the largest plateaus in tilt (coming from the most relevant cosines with minimal $n$ for a given $|k|$ ) will be those corresponding to $n \sim|k|^{-1}$.

Systems with $\mathbf{k}_{0}$ sufficiently near each of these values will be pinned and form a "plateau" in $\mathbf{k}$. How wide is this plateau? Let us suppose the putative tilt $\mathbf{k}_{0} \approx \mathbf{k}$. By scaling, the correlation length $\xi \sim 1 /\left|k_{0}\right| \sim n \gg 1 \mathrm{In}$ this situation, we must account for the renormalization of the relevant cosine term by the fluctuations on scales less than $\xi$. From standard renormalization group methods, 
one expects the renormalized coefficient

$$
\tilde{\lambda}_{n} \sim \lambda_{n} \xi^{-n^{2} \Delta}
$$

where $\Delta=\pi /(2 \sqrt{K})$ is the scaling dimension of the onemonopole term. Note that, from this reasoning, the $\tilde{\lambda}_{n}$ decrease extremely rapidly with $n$, hence from the above argument, incommensurate phases are possible.

In addition, for large $\xi$, the renormalized stiffness is small, $\tilde{\rho} \sim \rho / \xi^{2}$. On scales longer than $\xi$, the $\chi$ field is essentially non-fluctuating, so further renormalization can be neglected. The criteria for the system to be pinned at $\mathbf{k}$ is then $\tilde{\lambda}_{n} \gtrsim \tilde{\rho}|\delta k|^{2}$. Hence the width of the plateau is

$$
|\delta k| \lesssim \sqrt{\frac{\lambda_{n}}{\rho}} \xi^{1-n^{2} \Delta / 2}
$$

Clearly these commensurate plateaus are very narrow near the Lifshitz point. Similar estimates were obtained in Ref. 8 .

Each of the commensurate VBS phases breaks the discrete translational symmetry of the lattice, and thus must undergo a symmetry-restoring transition as temperature is increased. At non-zero temperature $T$, these states will be truncated to those commensurate VBS phases whose $T_{c}^{\prime} s$ are larger than $T$. In particular, consider a commensurately tilted VBS state driven by the $n$-monopole fugacity in the "center" of its plateau, i.e. for $\mathbf{k}=\mathbf{k}_{0}$. In this case, we may take $\delta \mathbf{k}=0$ in Eq. (34), and the system is described simply by a commensurate sine-Gordon model. At $T>0$, we may neglect all but the zero Matsubara frequency mode and set $\partial_{\tau} \chi=0$, encompassing the quantum effects by using the renormalized parameters $\tilde{\rho}, \tilde{\lambda}_{n}$ above. The symmetry-restoring transition is simply the roughening transition of this classical two dimensional sine-Gordon model, which has

$$
k_{B} T_{c}=\frac{2 \tilde{\rho}}{\pi n^{2}} \sim \frac{1}{n^{2} \xi^{2}} .
$$

For the strongest plateaus, recall $\xi \sim 1 /|k|$ and $n \sim 1 /|k|$, hence one has for these plateaus $k_{B} T_{c} \sim|k|^{4}$. The other plateaus have even smaller critical temperatures. Since all the critical temperatures vanish rapidly as $|k| \rightarrow 0$, the infinite set of plateaus in the devil's staircase is replaced by a finite subset at any non-zero temperature.

One may also estimate the excitation gap in the associated commensurate VBS phase, simply by expanding the sine-Gordon term to produce a $(\delta \chi)^{2}$ "mass" term. This gives a gap $E_{g}^{V B S} \sim n \sqrt{\tilde{\lambda}_{n}} \sim n \sqrt{\lambda_{n}} \xi^{-n^{2} \Delta / 2}$. It may be somewhat surprising that a state with an exponentially small gap can have such a relatively high critical temperature (power law in $1 / n$ from Eq. (38) ). The physics of this is that the excitation with an exponentially small gap consists of only small fluctuations of $\delta \chi$ (and hence the gauge electric field) which do not perturb the longrange order of the VBS state. Indeed, since the VBS phases are states of discrete broken symmetry, the excitations which do disturb this order by connecting the different symmetry-related ground states are "droplet"like. In the sine-Gordon language, such a droplet may be thought of as a domain wall in $\delta \chi$, which is wrapped around to form a compact "island" inside which $\delta \chi$ is shifted by $\pm 2 \pi / n$. In particular, the proliferation of such thermally excited droplets ultimately will "depin" the $\delta \chi$ field and destroy the VBS order above some temperature. The minimal radius of such an island is the domain wall width itself (since this is larger than the other natural cutoff, the correlation length). The energy for such a droplet is therefore given by integrating the exponentially small sine-Gordon term term over an exponentially large area of the size of the domain width (in reality there is also a comparable contribution from the $\tilde{\rho}$ term). These two factors compensate to give the relatively large energy determining $T_{c}$. The upshot of this argument is that the states with exponentially small excitation gap near the zero tilt QCP are not associated with the typical classical droplet excitations of a VBS state, but rather are evidence of the gapless photon mode obtaining precisely at the QCP.

A comment on the above discussion is in order. Within the sine-Gordon treatment, at any temperature above the "roughening" temperature, $\delta \chi$ behaves as a free scalar field, and vertex operators $\exp 2 \pi i n \delta \chi$ exhibit power law correlations. Ultimately, this can be tracked down as an artifact of the pure gauge theory. In particular, any matter fields included in the model, even gapped ones, correspond as discussed earlier to vortices in the $\chi$ (or $\delta \chi)$ field. At sufficiently high temperatures, these vortices will certainly unbind. However, for $n>4$, it is known that such sine-Gordon theories exhibit a "floating" phase in which power law correlations persist, above the roughening temperature $T_{c}$ and below the KosterlitzThouless temperature $T_{K T}$ above which vortices unbind. Here $k_{B} T_{K T}=\tilde{\rho} /(8 \pi)$. Note that one has then

$$
\frac{T_{K T}}{T_{c}}=\left(\frac{n}{4}\right)^{2}, \quad \text { for } n>4 .
$$

Hence the VBS phases very close to the Lifshitz point will have long-range VBS order only at very low temperatures $T<T_{c} \sim|k|^{4}$ but quasi-long-range VBS order up to much higher temperatures $T<T_{K T} \sim|k|^{2}$. Moreover, in the region with quasi-long-range order, there are no plateaus in the tilt.

In gauge theory language the roughening transition at $T_{c}$ may be associated with the thermally driven deconfinement transition of pure gauge theories. Indeed, electric field correlators in this rough phase (gradients of the height field) fall off as the inverse square of the distance - so this phase may be thought of as a 'thermal Coulomb phase'. The gauge charged spinons are logarithmically interacting in this phase and are bound into gauge neutral pairs until they ionize at $T_{K T}$ leading to a plasma of gauge charge that destroys the long range electric field correlations of the thermal Coulomb (or rough) 
phase. This transition is also studied in detail in ${ }^{4}$, in theories with a noncompact gauge field and $\mathrm{SU}(2)$ symmetric spion fields. While the thermal deconfinement transition of gauge theories is generally expected only in the absence of matter with unit gauge charge (spinons), in two spatial dimensions the logarithmic form of the Coulomb interactions is strong enough to bind the spinons into gauge neutral pairs and hence the transition survives the inclusion of spinons.

\section{THE SINGLE LAYER HONEYCOMB LATTICE}

In contrast to the situation analysed above for the bilayer honeycomb aniferromagnet, in a single layer the appropriate lattice sine Gordon model has non-zero offsets $\alpha_{r}$ for the $\chi$ fields on the three sublattices of the dual triangular lattice. Thus as explained in Ref. 8 the $\chi_{r} \rightarrow-\chi_{r}$ transformation becomes a symmetry only when combined with inversion or a $\pi / 3$ rotation. This then leads to the possibility of a cubic invariant in the sine Gordon action which drives the transition first order.

It is interesting to ask about the situation with spin$3 / 2$ antiferromagnets on a single layer honeycomb lattice. In this case there are no offsets for the $\chi_{r}$ in the sine Gordon description of the paramagnetic phase. Nevertheless $\chi_{r} \rightarrow-\chi_{r}$ symmetry (without inversion or $\pi / 3$ rotation) is not expected as an exact symmetry of the action. This is not required by any of the microscopic symmetries of the underlying lattice spin model. Hence we expect that a cubic term will still be allowed in the field theory and a first order transition will result.

\section{THE SQUARE LATTICE}

We now perform the same analysis for the transition in the case of the spin one half quantum antiferromagnet on the square lattice. In contrast to the situation on the honeycomb lattice, we will find no generic continuous transition between VBS states (in both single and bilayer cases).

As before we consider a generic continuum theory that is consistent with all the lattice and internal symmetries. For the square lattice this takes the form

$$
\begin{aligned}
S & =S_{c}+S_{i n t}+S_{m o n} \\
S_{c} & =\frac{1}{2} \int\left(\partial_{\tau} \chi\right)^{2}+K\left[\left|\nabla^{2} \chi\right|^{2}+4 \sigma\left(\partial_{x}^{2} \chi\right)\left(\partial_{y}^{2} \chi\right)\right]( \\
S_{\text {int }} & =\int \frac{u}{4}\left[\left(\partial_{x} \chi\right)^{4}+\left(\partial_{y} \chi\right)^{4}\right]+\frac{v}{2}\left(\partial_{x} \chi\right)^{2}\left(\partial_{y} \chi\right)^{2}
\end{aligned}
$$

with $\sigma>-1$. The isotropic point corresponds to $\sigma=0$ and $u=v$. The last term $\left(S_{m o n}\right)$ represents quadrupled monopole events. Note in particular the presence of the couplings $\sigma, v$ which are allowed by the square lattice symmetry. Consider the critical theory given by the quadratic piece of the action $S_{c}$ and determine its stability against the inclusion to small monopole tunneling. In this case we need to consider quadrupled monopoles, as discussed in section II and once again it is possible to find a range of $K, \sigma$ for which monopole tunneling is irrelevant.

Next, one must consider the stability of the Gaussian fixed point described by $S_{c}$ to turning on the quartic interaction (42). Again, we perform a one loop renormalization group analysis to determine the fate of these couplings. The result of integrating the high wavevector modes $\Lambda(1-d l)<|k|<\Lambda$ and rescaling, are the following $\mathrm{RG}$ equations:

$$
\begin{aligned}
& \frac{d U}{d l}=-3\left[9 \alpha U^{2}+2 U V+\alpha V^{2}\right], \\
& \frac{d V}{d l}=-9\left[U^{2}+2 \alpha U V+V^{2}\right],
\end{aligned}
$$

where we have used the scaled variables $U=u \mathcal{A}^{-1}, V=$ $v \mathcal{A}^{-1}$, and the scale factor $\mathcal{A}=(1+\sigma+\sqrt{1+\sigma}) 32 \pi K^{\frac{3}{2}}$. At the isotropic point, these equations are identical to (32), and preserve $u=v$. The anisotropy of the quadratic part of the action is present in the parameter $\alpha$, which is unity at the isotropic point but otherwise is given by:

$$
\alpha=\frac{1}{3}\left[\frac{2 \sigma+1-\sqrt{1+\sigma}}{\sqrt{1+\sigma}-1}\right]
$$

thus $\alpha \in[1 / 3, \infty)$.

We now analyze the RG equations (43 44), and show that they imply that the critical Gaussian theory $S_{c}$ is generally unstable in the presence of the two quartic operators. If these were stable critical points, then there should be a region in the $u, v$ plane where the flows end up at the origin. However we will show that there is only a single line in the entire $u, v$ plane, where the couplings flow into the origin. This implies that stability is only attained on a set of measure zero points. For a general choice of the quartic coupling, the flows run away to large negative values of $u$ suggesting a first order transition. In order to show this property of the RG equations (4344), we construct a function $E(U, V)$ that is invariant along any $\mathrm{RG}$ trajectory i.e. $\frac{d E}{d l}=0$. Contour lines of this function then represent the RG flows, and we will see that there is only a single contour connected to the origin. This function is most conveniently represented in terms of the rotated coordinates, $U_{+}=\alpha U+V, U_{-}=U-\alpha V$. Then,

$$
E=\frac{U_{-}^{3}}{\left(\alpha^{2}+3\right) U_{-}^{2}+4 \alpha U_{+} U_{-}+\left(3 \alpha^{2}+1\right) U_{+}^{2}},
$$

which can be checked to be invariant under the RG flows. It may be seen that the origin must correspond to $E=$ 0 , by approaching it in any direction. The only other points for which the invariant function vanishes is the line $U_{-}=0$, which corresponds to $u=\alpha v$. These are the only points that could flow to the origin. Apart from 
this set of measure zero, none of the other points in the $u, v$ plane ever reach the origin under the RG flows but rather flow to regions with large negative values of $u$. Thus, for the case with square symmetry, the Gaussian critical theory is generically unstable - the flows suggest a first order transition in the absence of special fine-tuning.

\section{RK POINTS}

The quantum dimer model Hamiltonians studied in 7 were shown to have a special point - the RK point - at which the wavefunction is an equal superposition of all dimer configurations. Equal time correlation functions can then be evaluated from the classical dimer model, which has been extensively studied 20 . In this section we address how those results fit into the framework discussed here - for the case of the single layer bipartite lattices. For example we may ask in the case of the honeycomb lattice where a transition through a multicritical point can obtain by tuning two parameters, whether the RK wavefunction corresponds to the the ground state wave function of any of these fixed points. In fact, we will conclude that while the RK points in both the square and the honeycomb lattice cases can reproduce critical properties of some point on the line of fixed points obtained after tuning a few parameters in the generic models, they represent very special multicritical points in terms of their position in the phase diagram of these generic models. Thus accessing these points requires fine tuning an infinite number of independent operators, which do not affect the critical properties but change the phase structure in the immediate vicinity of the point (dangerously irrelevant operators). This fine tuned nature of the RK point can immediately be seen by noting that at the RK point, the ground state wavefunction in each winding number (tilt) sector has exactly the same energy. Reproducing this democratic treatment of all winding number sectors within a height model representation of the RK point will require tuning an infinite number of parameters to zero in the bare Hamiltonian - even though they may be associated with operators that are (dangerously) irrelevant at the critical point.

If the RK point requires fine tuning infinitely many parameters, one may ask how it is accessed so readily in the quantum dimer model. The reason is that the dimer model usually contains only single plaquette terms, where the RK point is accessed by tuning just one parameter. However, including processes that involve several plaquettes will require tuning increasingly larger number of parameters to obtain the equal dimer superposition of the RK point. Generically the dimer representation of spin systems will include processes that involve arbitrarily large number of plaquettes. Although these decay with increasing size, they are generally non vanishing, and infinite fine tuning will be then be required to reach the RK point.

For the square lattice, the field theory that reproduces

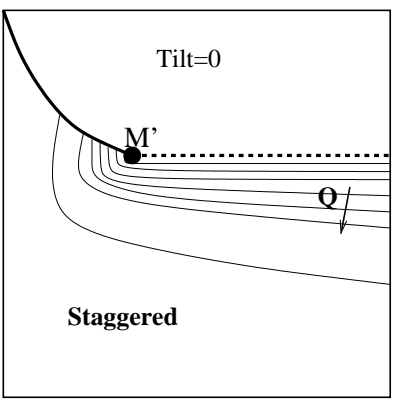

(a)

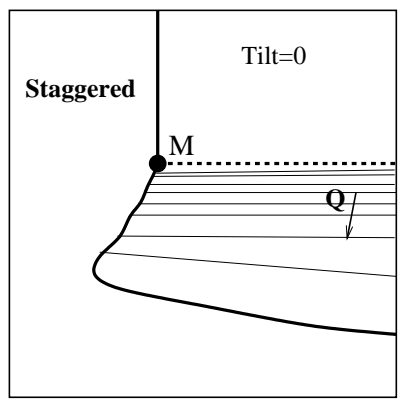

(b)

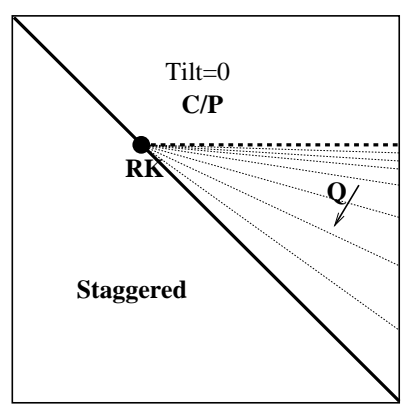

(c)

FIG. 2: Schematic depiction of the phase diagram of VBSs, the vertical axis in the plots is roughly the the parameter $\rho$ and the staggered state has the maximum tilt. The generic phase diagrams expected for the bilayer honeycomb lattice are shown in (a) and (b) (and also of the single layer honeycomb lattice after tuning one parameter, the cubic term, to zero). (a) The continuous transition (shown with the dashed line) is from a zero tilt phase to a region where the tilt $(\mathbf{Q})$ exhibits a a devil's staircase structure, contours of equal tilt (thin solid lines) are shown. The critical line ends in a multicritical point $M^{\prime}$ beyond which the transition is first order (solid line). The horizontal axis here may be thought of as the coefficient of the quartic term. (b) An alternate scenario, there is again a continuous transition to a region with the devil's staircase structure for the tilt. Here however, there is a multicritical point $M$ that is adjacent to the staggered state which could control a zero tilt to staggered state transition. The horizontal axis here may be thought of as the energy cost of the maximally tilted (staggered) state. (c) The RK point for the single layer square/honeycomb lattice - an infinite number of parameters need to be tuned to access this plane. Exact degeneracy of the different winding number sector ground states implies that states with arbitrary tilt lie infinitesimally close to the RK point as shown.

asymptotic properties of the RK point was studied by Henley ${ }^{5}$. It was found there that a quadratic action (40) with $K=\pi^{2} / 4, \sigma=0$ gives the long distance dimer correlations at the RK point (amusingly this is the parameter value at which the two monopole insertion operator is marginal). The fact that the ground states in all winding number sectors are degenerate at the RK point implies that it is possible to access states with arbitrary values of the tilt my moving infinitesimally away from the RK 
point. As a result, the topology of the phase diagram near the RK point is as shown in figure (2). An infinite number of parameters have been set to zero to access this plane of the phase diagram. While the continuous transition from the zero tilt state leads to the devil's staircase of tilted states, a direct transition from a zero tilt state to the staggered state can be made by crossing the RK point. Thus the RK point terminates the critical line, and sits on a line of first order transitions. The special nature of the RK point, even as a multicritical point, can be seen by comparing it to the generic multicritical points of the bilayer honeycomb lattice (which require tuning of two parameters to reach) denoted $M^{\prime}, M$ in figures 2a,b. (These can also be thought of as higher order multicritical points in the single layer honeycomb model, which will require fine tuning of an additional parameter to reach the plane depicted in the diagrams.)In the first scenario depicted in figure 20, the multicritical point $M^{\prime}$ terminates both the first order transition line and the critical line, but it does not allow for a direct transition from the zero tilt state to the staggered state. In the other scenario figure (20) the generic multicritical point $M$ does allow for a direct transition from a zero tilt state to the staggered state. However, it is fundamentally distinct from the RK point, as can be seend from the difference in topology of the phase structure around the $M$ and RK points, which may be characterized as follows. We ask what phases may be accessed from these points by a small change of bare parameters. For the generic multicritical point $M$, the staggered state, the zero tilt state, and a state with infinitesimal tilt can be accessed. For the RK point however, besides the staggered and zero tilt state, states with arbitrary values of the tilt can also be accessed in this manner, as shown in the figure. This follows from the exact degeneracy of ground states in different winding number sectors at the RK point.

\section{CONCLUSIONS}

In this paper we have shown that at least in certain instances there are direct second order transitions between distinct valence bond solid phases. Deconfinement obtains at the critical point though both phases are conventional and confined. More precisely the critical theory may be viewed as a gapless $U(1)$ gauge theory with irrelevant instantons. The spin carrying excitations in either phase are gapped spin-1 magnons or their composites. Right at the critical point however the spin gap does not close but the magnons decay into (gapped) spin-1/2 spinons.

All of this structure is very similar to the other examples of 'deconfined' quantum criticality studied in Ref ${ }^{2}$. However there are some interesting differences in the details. First the critical points discussed in the present paper are controlled by a critical fixed line with continuously varying exponents. Second (upto a marginally irrelevant non-linear operator) all points on this fixed line have a simple free field description. The emergence of a (topological) global $U(1)$ symmetry that seems generic to deconfined quantum criticality obtains for the transitions in this paper as well. However the free field description implies an enormous number of further emergent symmetries which are specific to these transitions. Third, the dynamic scaling exponent $z$ is 2 in the present example (compared to $z=1$ at the Neel-VBS transition). Finally as detailed in Section IIC there is rich and interesting structure with an infinite number of transitions on one side of the deconfined critical point.

One off-shoot of these results is a clarification of the place of the solvable RK point of quantum dimer models in a more general context of phase transitions in quantum magnets. We find that the RK point corresponds to a special multicritical point.

We also showed that these interesting phase transitions are best realized in bilayer spin- $1 / 2$ honeycomb lattice quantum antiferromagnets. It would be interesting for numerical work to explore specific spin models on such bilayers where these transitions can be accessed.

Several extensions of our results are possible. It should be possible to examine the role of various perturbing fields at the critical point as well as the effects of finite temperature. It should also be readily possible to examine transitions between different VBS phases in higher spin quantum magnets. We leave these for future work.

Since the original submission of an electronic preprint of this work, Ref. 8 appeared which considered some of the same questions especially those regarding the single layered systems discussed here. They correctly pointed out the first order nature of the transition on the single layer honeycomb lattice, and the 'incompleteness' of the devil's staircase - which were the points of disagreement with the earlier version of this work. These points have been corrected and briefly mentioned in the present work. However in contrast to Ref. 8 we have focused here on the case of the bilayer honycomb quantum magnet where a generically continuous transition between VBS states $i s$ realised, and have studied some of the interesting properties of this generic quantum critical point.

\section{ACKNOWLEDGEMENTS}

This work was sparked by conversations with S. Sondhi and Y.B. Kim. We are particularly grateful to the former for sharing his insights on quantum dimer models and the physics of the RK point. TS would like to thank O. Motrunich for a very useful prior collaboration on related field theories. We also thank E. Fradkin and S. Sachdev for useful discussions, and D. Huse and C. Henley for pointing out some errors in a previous version of the manuscript. This research was generously supported by the National Science Foundation under grants DMR-0308945 (T.S.), DMR-9985255 (L.B.). We would also like to acknowledges funding from the NEC Corporation (T.S.), the Packard Foundation (L.B.), the Alfred 
P. Sloan Foundation (T.S., L.B.), a Pappalardo Fellowship (A.V.) and an award from The Research Corporation (T.S.).

\section{APPENDIX A: CRITICAL GAUGE THEORY}

In this Appendix we will explicitly display the form of the continuum action of the non-compact $U(1)$ gauge theory that corresponds to the critical sine Gordon theory. A general continuum Hamiltonian for a non-compact $U(1)$ gauge theory in $D=2+1$ dimensions takes the form

$$
H=\int d^{2} x \rho|\mathbf{E}|^{2}+K|\nabla \times \mathbf{E}|^{2}+B^{2}+\cdots
$$

together with the Gauss law constraint

$$
\nabla \cdot \mathbf{E}=0
$$

Here $\mathbf{E}$ refers to the electric field while $B=\hat{\mathbf{z}} \cdot \boldsymbol{\nabla} \times \mathbf{A}=$ $\epsilon_{i j} \partial_{i} A_{j}$ is the magnetic field. We work in the Coulomb gauge $\boldsymbol{\nabla} \cdot \mathbf{A}=0$. As usual the components of the electric field $E_{i}(i=x, y)$ and the (transverse) components $a_{i}$ of the vector potential are canonically conjugate:

$$
\left[E_{i}(\mathbf{x}), A_{j}\left(\mathbf{x}^{\prime}\right)\right]=-i P_{i j}\left(\mathbf{x}-\mathbf{x}^{\prime}\right),
$$

where $P_{i j}$ has Fourier components $\delta_{i j}-\frac{k_{i} k_{j}}{k^{2}}$ and projects out the transverse component.

The Gauss law constraint may be solved by writing

$$
E_{i}=\epsilon_{i j} \partial_{j} \chi
$$

where $\chi$ is a scalar field. Assume that the commutator of $\chi$ and $A_{j}$ takes the form

$$
\left[\chi(\mathbf{x}), A_{j}\left(\mathbf{x}^{\prime}\right)\right]=i f_{j}\left(\mathbf{x}-\mathbf{x}^{\prime}\right)
$$

The correct commutator between $\mathbf{E}$ and $\mathbf{A}$ is reproduced if we impose

$$
\epsilon_{i k} \partial_{k} f_{j}\left(\mathbf{x}-\mathbf{x}^{\prime}\right)=-P_{i j}\left(\mathbf{x}-\mathbf{x}^{\prime}\right)
$$

This then implies the commutator

$$
\left[\chi(\mathbf{x}), B\left(\mathbf{x}^{\prime}\right)\right]=i \delta\left(\mathbf{x}-\mathbf{x}^{\prime}\right)
$$

so that the magnetic field $B$ is conjugate to $\chi$. The Hamiltonian may now be rewritten

$$
H=\int d^{2} x \rho|\nabla \chi|^{2}+K\left(\nabla^{2} \chi\right)^{2}+B^{2}
$$

As $B$ and $\chi$ are canonically conjugate we reproduce the continuum free field action of the sine Gordon theory. Remembering that the critical theory has $\rho=0$ we can immediately read off from Eqn. A1 the continuum gauge theory Hamiltonian that describes the critical point. This is readily diagonalized explicitly to find a quadratic dispersing photon.

Amusingly, at $\rho=0$, the Hamiltonian A8 exhibits a kind of self 'duality', obtained by exchanging the roles of $B$ and $\nabla^{2} \chi$. That is, if we introduce the field $\phi$ such that $B=\frac{\nabla^{2} \phi}{\sqrt{K}}$, and its conjugate field $\Pi_{\phi}=-\sqrt{K} \nabla^{2} \chi$, then one obtains the same critical Hamiltonian A8 with $\rho=0)$ but with $K \rightarrow 1 / K$ and $(B, \chi) \rightarrow\left(\Pi_{\phi}, \phi\right)$.
1 J. A. Hertz, Phys. Rev. B 14, 1165 (1976).

2 T. Senthil, A. Vishwanath, L. Balents, S. Sachdev, and M. P. A. Fisher, cond-mat/0311326

3 T. Senthil, L. Balents, S. Sachdev, A. Vishwanath, and M. P. A. Fisher, cond-mat/0312617

4 O. Motrunich and A. Vishwanath cond-mat/0311222

${ }^{5}$ C.L. Henley, J. Stat. Phys. 89, 483 (1997). C.L. Henley, cond-mat/ 0311345

6 R. Moessner, S. L. Sondhi, and E. Fradkin, Phys. Rev. B 65, 024504 (2002).

7 D. S. Rokhsar and S. A. Kivelson, Phys. Rev. Lett. 61, 2376 (1988).

8 E. Fradkin, D.A. Huse, R. Moessner, V. Oganesyan, and S. Sondhi, cond-mat/0311353

9 G. Grinstein, Phys. Rev. B 23, 4615 (1981).

10 N. Read and S. Sachdev, Phys. Rev. Lett. 62, 1694 (1989); N. Read and S. Sachdev, Phys. Rev. B 42, 4568 (1990).

11 E. Fradkin and S. A. Kivelson, Mod. Phys. Lett. B 4, 225 (1990); E. Fradkin, Field theories of Condensed Matter
Systems, Perseus Books (1991).

12 E. Ardonne, P. Fendley and E. Fradkin, cond-mat/ 0311466

13 C. Lannert, M. P. A. Fisher, and T. Senthil, Phys. Rev. B 63, 134510 (2001).

14 A. M. Polyakov, Gauge Fields and Strings, Hardwood Academic Publishers (1987).

15 For a recent review see S. Sachdev, Annales Henri Poincare 4, 554 (2003).

16 F. D. M. Haldane, Phys. Rev. Lett. 61, 1029 (1988).

17 S. Sachdev and K. Park, Annals of Physics, N.Y. 298, 58 (2002).

18 This is most readily seen in a $C P^{1}$ description in which a skyrmion corresponds to $2 \pi$ flux of the $U(1)$ gauge field seen by the spinons.

19 S. Aubry and P. Y. Le Daeron, Physica D 8, 381 (1983).

20 M. E. Fisher and J. Stephenson, Phys. Rev. 132, 1411 (1963). 